\title{
Corrigendum
}

\section{Corrigendum to "Overexpression of GRK3, Promoting Tumor Proliferation, Is Predictive of Poor Prognosis in Colon Cancer"}

\author{
Tao Jiang, ${ }^{1}$ Chun Yang, ${ }^{1}$ Liyuan $\mathrm{Ma},{ }^{2}$ Zehua $\mathrm{Wu},{ }^{3}$ Ling Ye, ${ }^{4}$ Xiaoqiang $\mathrm{Ma},{ }^{1} \mathrm{Hai}$ Li, ${ }^{1}$ \\ Junwei Fan $\left(\mathbb{C}^{4},{ }^{4}\right.$ and Yinxue Yang $\mathbb{1}^{1}$ \\ ${ }^{1}$ Department of Anal-Colorectal Surgery, General Hospital of Ningxia Medical University, 804 South Shengli Road, \\ Yinchuan 750004, China \\ ${ }^{2}$ Department of Ultrasound, General Hospital of Ningxia Medical University, 804 South Shengli Road, Yinchuan 750004, China \\ ${ }^{3}$ Department of Hepatobiliary Surgery, The Affiliated Hospital of Medical College, Qingdao University, Qingdao 266000, China \\ ${ }^{4}$ Department of General Surgery, Shanghai Jiao Tong University Affiliated First People's Hospital, 85 Wujin Road, \\ Shanghai 200080, China
}

Correspondence should be addressed to Junwei Fan; doctorjunwei@gmail.com and Yinxue Yang; 18895007660@163.com

Received 21 September 2020; Accepted 21 September 2020; Published 23 October 2020

Copyright ( 2020 Tao Jiang et al. This is an open access article distributed under the Creative Commons Attribution License, which permits unrestricted use, distribution, and reproduction in any medium, provided the original work is properly cited.

In the article titled "Overexpression of GRK3, Promoting Tumor Proliferation, Is Predictive of Poor Prognosis in Colon Cancer" [1], there was an error in the beta-actin forward primer. It was written as CGGGAAATGTGCGTGAC, and it should be corrected to CGGGAAATCGTGCGTGAC.

\section{References}

[1] T. Jiang, C. Yang, L. Ma et al., "Overexpression of GRK3, promoting tumor proliferation, is predictive of poor prognosis in colon cancer," Disease Markers, vol. 2017, Article ID 1202710, 11 pages, 2017. 